\title{
An axial gauge ansatz for higher spin theories
}

\section{Steven S. Gubser and Wei Song}

Joseph Henry Laboratories, Princeton University, Princeton, NJ 08544, U.S.A.

E-mail: ssgubser@Princeton.edu, wsong@math.tsinghua.edu.cn

ABSTRACT: We present an ansatz which makes the equations of motion more tractable for the simplest of Vasiliev's four-dimensional higher spin theories. The ansatz is similar to axial gauge in electromagnetism. We present a broad class of solutions in the gauge where the spatial connection vanishes, and we discuss the lift of one of these solutions to a full spacetime solution via a gauge transformation.

Keywords: Higher Spin Symmetry, AdS-CFT Correspondence

ARXIV EPRINT: 1405.7045 


\section{Contents}

1 Introduction 1

2 The equations of motion $\quad 2$

$\begin{array}{lll}3 & \text { The ansatz } & 4\end{array}$

4 Gauge transformations and a mass deformation $\quad 6$

$\begin{array}{ll}4.1 \text { The spacetime connection } & 6\end{array}$

$\begin{array}{lll}4.2 & \text { An example } & 7\end{array}$

5 Conclusions $\quad 9$

\section{Introduction}

Vasiliev's higher spin theories in four dimensions $[1,2]$ are relatively simple theories involving infinitely many fields, all with integer spin. The full non-linear equations of motion are known, and the simplest solution to them is $A d S_{4}$. Some additional solutions of the equations (2.2) are known: see for example [3-5]. Finding exact solutions is challenging because the equations of motion are non-linear and involve a non-local star product in the oscillator variables. But a broader set of exact solutions is highly desirable in order to advance our understanding of classical higher spin theory beyond perturbation theory. The aim of this paper is to introduce a new class of exact solutions. In one subcase of our construction, the solutions are parametrized by an arbitrary function of three variables, making it a remarkably large class of solutions.

Vasiliev's equations involve auxiliary, bosonic, spinorial variables $z^{\alpha}$, and one of the equations of motion takes the form $f_{z^{1} z^{2}}=-p(b * K)$, where $f_{z^{1} z^{2}}$ is like a Yang-Mills field strength, $b * K$ is covariantly constant in the adjoint representation, and $p$ is a phase - for our purposes, either 1 or $i$. The equation $f_{z^{1} z^{2}}=-p(b * K)$ is formally similar to having a magnetic field in two dimensions: $\partial_{1} A_{2}-\partial_{2} A_{1}=B_{12}$. A standard strategy is to set $A_{1}=0$ as a gauge choice and then solve for $A_{2}$ in terms of $B_{12}$. This is axial gauge. We are going to make an analogous ansatz, namely $s_{1}=0=\bar{s}_{1}$ where $s_{\alpha}$ is the spinorial part of the gauge potential with field strength $f_{z^{1} z^{2}}$, and $\bar{s}_{\dot{\alpha}}$ corresponds to a conjugate field strength $f_{\bar{z}^{\mathrm{i}} \bar{z}^{2}}$. This choice appears to be as innocuous as the choice of axial gauge; however, our overall ansatz is more restrictive than just a gauge choice.

Setting $s_{1}=\bar{s}_{\dot{1}}=0$ removes some star-(anti)-commutators from the equations of motion, so that some components of these equations become linear. After solving these linear equations (in a gauge where the spacetime components of the higher spin connection vanish), we find that the non-linear equations reduce to quadratic constraints on the ansatz. 
These quadratic constraints have many solutions, especially in a particular case where a principle of superposition operates, allowing us to construct solutions labeled by the aforementioned arbitrary function of three variables. Related strategies have been pursued in previous work $[4,6]$; a common thread is rendering the equation for $f_{z^{1} z^{2}}$ effectively linear.

The structure of the rest of the paper is as follows. For the sake of a self-contained presentation, we review in section 2 the equations of motion of the higher-spin theories that we are going to solve. In section 3 we explain in detail the ansatz and show some examples of solutions. The treatment of this section relies entirely on a gauge where the spacetime components of the connection vanish, also described as the $Z$-space approach in [3]. In section 4 we discuss how solutions of the type obtained in the previous section can be lifted via a gauge transformation to full spacetime solutions. We focus on a particular route to the Poincaré patch of $A d S_{4}$, but a different gauge transformation would lead to global $A d S_{4}$. An example presented in section 4.2 leads to an exact solution of the Vasiliev equations in which the spatial part of the higher spin connection is the same as in $A d S_{4}$ and the scalar takes a form which, in the linearized theory, is associated with a massive deformation of the $O(N)$ model. It is tempting to identify the exact solution as dual to the massive $O(N)$ model; however, we caution that the explicit breaking of Lorentz symmetry inherent in our ansatz complicates this interpretation.

\section{The equations of motion}

The equations of motion of Vasiliev's higher spin theories in four dimensions [1,2] can be stated in terms of a gauge field

$$
A=W_{\mu} d x^{\mu}+S_{\alpha} d z^{\alpha}+\bar{S}_{\dot{\alpha}} d \bar{z}^{\dot{\alpha}}
$$

and a scalar field $B$ : following the conventions of [7], one writes

$$
\begin{aligned}
F & \equiv d A+A * A=p(B * K) d z^{2}+\bar{p}(B * \bar{K}) d \bar{z}^{2} \\
D B & \equiv d B+A * B-B * \pi(A)=0,
\end{aligned}
$$

where $K, \bar{K}, \pi, d z^{2}, d \bar{z}^{2}$, and $*$ are defined in the paragraphs below. The phase $p$ is 1 for the so-called type A theory, dual to the $O(N)$ model [8] and $i$ for type B, dual to the Gross-Neveu model [9]; correspondingly, $\bar{p}=1$ or $-i$.

The components of $A$, and also $B$, are functions of the usual four bosonic coordinates $x^{\mu}$ together with spinorial oscillator coordinates (also bosonic) $Y^{A}=\left(y^{\alpha}, \bar{y}^{\dot{\alpha}}\right)$ and $Z^{A}=$ $\left(z^{\alpha}, \bar{z}^{\dot{\alpha}}\right)$, where $\alpha$ and $\dot{\alpha}$ are doublet indices for the irreducible spinor representations of $\mathrm{SO}(3,1)$. The coordinates $Y^{A}$ do not participate in the differential structure of the theory: in other words, the exterior derivative $d$ acts only on $x^{\mu}$ and $Z^{A}$, and we never encounter one-forms $d Y^{A}$. $A$ and $B$ admit series expansions in $Y^{A}$ and $Z^{A}$. Roughly speaking, the metric and spin connection come from the terms in $A$ that are quadratic in the $Y^{A}$ coordinates, while the part of $B$ which depends only on the $x^{\mu}$ is identified as a scalar field.

To formulate the equations, one uses an associative star product, defined by

$$
f(Y, Z) * g(Y, Z)=\mathcal{N} \int d^{4} u d^{4} v f(Y+U, Z+U) g(Y+V, Z-V) e^{U^{A} V_{A}},
$$


where the normalization factor $\mathcal{N}$ is such that $f * 1=f$. Indices are raised and lowered according to

$$
U^{A}=\Omega^{A B} U_{B} \quad U_{A}=U^{B} \Omega_{B A} .
$$

Here

$$
\Omega_{A B}=\Omega^{A B}=\left(\begin{array}{cc}
\epsilon_{\alpha \beta} & 0 \\
0 & \epsilon_{\dot{\alpha} \dot{\beta}}
\end{array}\right)
$$

and

$$
\epsilon_{\alpha \beta}=\epsilon^{\alpha \beta}=\epsilon_{\dot{\alpha} \dot{\beta}}=\epsilon^{\dot{\alpha} \dot{\beta}}=\left(\begin{array}{cc}
0 & 1 \\
-1 & 0
\end{array}\right) .
$$

The star product is associative, and

$$
\begin{array}{ll}
Y^{A} * Y^{B}=Y^{A} Y^{B}+\Omega^{A B} & Z^{A} * Z^{B}=Z^{A} Z^{B}-\Omega^{A B} \\
Y^{A} * Z^{B}=Y^{A} Z^{B}-\Omega^{A B} & Z^{A} * Y^{B}=Z^{A} Y^{B}+\Omega^{A B} .
\end{array}
$$

The Kleinians

$$
K \equiv e^{z^{\alpha} y_{\alpha}} \quad \bar{K} \equiv e^{\bar{z}^{\dot{\alpha}} \bar{y} \dot{\alpha}}
$$

satisfy $K * K=\bar{K} * \bar{K}=1$, and also

$$
f(y, \bar{y} ; z, \bar{z}) * K=K f(-z, \bar{y} ;-y, \bar{z}) \quad K * f(y, \bar{y} ; z, \bar{z})=K f(z, \bar{y} ; y, \bar{z}) .
$$

The map $\pi$, and a closely related map $\bar{\pi}$, are defined by

$$
\begin{aligned}
& \pi(f(y, \bar{y} ; z, \bar{z} ; d z, d \bar{z}))=f(-y, \bar{y} ;-z, \bar{z} ;-d z, d \bar{z}) \\
& \bar{\pi}(f(y, \bar{y} ; z, \bar{z} ; d z, d \bar{z}))=f(y,-\bar{y} ; z,-\bar{z} ; d z,-d \bar{z}) .
\end{aligned}
$$

For zero-forms (i.e. cases where $f$ doesn't depend on $d z$ or $d \bar{z}$ ), we have $\pi(f)=K * f * K$ as a consequence of (2.9). We also define

$$
d z^{2}=\frac{1}{2} d z^{\alpha} \wedge d z_{\alpha}=-d z^{1} \wedge d z^{2} \quad d \bar{z}^{2}=\frac{1}{2} d \bar{z}^{\dot{\alpha}} \wedge d \bar{z}_{\dot{\alpha}}=-d \bar{z}^{\dot{1}} \wedge d \bar{z}^{\dot{2}} .
$$

All definitions needed in (2.2) are now explicit.

Passing locally to a gauge where the higher spin spacetime connection $w$ vanishes, the higher spin equations take the form

$$
\begin{aligned}
d_{Z} s+s * s & =p(b * K) d z^{2}+\bar{p}(b * \bar{K}) d \bar{z}^{2} \\
d_{Z} b+s * b-b * \pi(s) & =0
\end{aligned}
$$

where $s=s_{\alpha} d z^{\alpha}+\bar{s}_{\dot{\alpha}} d \bar{z}^{\dot{\alpha}}$ is the spinorial part of the gauge field, and $b, s_{\alpha}$, and $\bar{s}_{\dot{\alpha}}$ are now functions only of $Y^{A}$ and $Z^{A}$. Dependence on $x^{\mu}$ is prevented by the $x^{\mu}$ components of the full equations of motion (2.2) in the $w=0$ gauge. By $d_{Z}$ we mean the exterior derivative with respect to only the $Z^{A}$ variables; likewise, $d_{x}$ refers to the exterior derivative with respect to only the $x^{\mu}$ variables. We use lowercase $b$ and $s$ in $w=0$ gauge so as to distinguish these quantities from their images in a more general gauge. 


\section{The ansatz}

In components, the equations (2.12) read

$$
\begin{aligned}
\frac{\partial s_{2}}{\partial z^{1}}-\frac{\partial s_{1}}{\partial z^{2}}+\left[s_{1}, s_{2}\right]_{*} & =-p(b * K) \\
\frac{\partial b}{\partial z^{\alpha}}+s_{\alpha} * b+b * \pi\left(s_{\alpha}\right) & =0 \\
\frac{\partial \bar{s}_{\dot{2}}}{\partial \bar{z}^{\dot{1}}}-\frac{\partial \bar{s}_{\dot{1}}}{\partial \bar{z}^{2}}+\left[\bar{s}_{\dot{1}}, \bar{s}_{\dot{2}}\right]_{*} & =-\bar{p}(b * \bar{K}) \\
\frac{\partial b}{\partial \bar{z}^{\dot{\alpha}}}+\bar{s}_{\dot{\alpha}} * b-b * \pi\left(\bar{s}_{\dot{\alpha}}\right) & =0 \\
\frac{\partial \bar{s}_{\dot{\beta}}}{\partial z^{\alpha}}-\frac{\partial s_{\alpha}}{\partial \bar{z}^{\dot{\beta}}}+\left[s_{\alpha}, \bar{s}_{\dot{\beta}}\right]_{*} & =0,
\end{aligned}
$$

where $[f, g]_{*}=f * g-g * f$. Let's assume

$$
s_{1}=0=\bar{s}_{\dot{1}} \quad \frac{\partial s_{2}}{\partial \bar{z}^{2}}=0=\frac{\partial \bar{s}_{\dot{2}}}{\partial z^{2}} \quad \frac{\partial b}{\partial Z^{A}}=0 .
$$

These choices are convenient because the equations (3.1) reduce to

$$
\begin{aligned}
& \frac{\partial s_{2}}{\partial z^{1}}=-p(b * K) \quad \frac{\partial \bar{s}_{\dot{2}}}{\partial \bar{z}^{\dot{1}}}=-\bar{p}(b * \bar{K}) \\
& \left\{s_{2}, b * K\right\}_{*}=0 \quad\left[\bar{s}_{\dot{2}}, b * K\right]_{*}=0 \quad\left[s_{2}, \bar{s}_{\dot{2}}\right]_{*}=0,
\end{aligned}
$$

where $\{f, g\}_{*}=f * g+g * f$. Given $b=b\left(Y^{A}\right)$, we can immediately solve the first two equations in (3.3):

$$
\begin{array}{llll}
s_{2}=\int_{0}^{1} d t \sigma_{2}(t) & \text { where } & \sigma_{2}(t)=-p z^{1}[b * K]_{z^{1} \rightarrow t z^{1}} \\
\bar{s}_{\dot{2}}=\int_{0}^{1} d \tilde{t} \bar{\sigma}_{\dot{2}}(\tilde{t}) & \text { where } & \bar{\sigma}_{\dot{2}}(\tilde{t})=-\bar{p} \bar{z}^{\mathrm{i}}[b * \bar{K}]_{\bar{z}^{\mathrm{i}} \rightarrow \tilde{t} \bar{z}^{\mathrm{i}}} .
\end{array}
$$

Note that the holomorphy conditions $\frac{\partial s_{2}}{\partial \bar{z}^{2}}=0=\frac{\partial \bar{s}_{\dot{2}}}{\partial z^{2}}$ which we assumed in (3.2) are automatically satisfied by (3.4). Starting with $b=b\left(Y^{A}\right)$ and extracting $S$ through an integration similar to (3.4) is a standard beginning to the perturbative approach of solving (2.12): see for example $[3,7]$. The assumptions (3.2) make this perturbative approach exact. However, the quadratic constraints in the second line of (3.3) must still be checked, and they do not hold for arbitrary functional forms $b\left(Y^{A}\right)$. Before we indicate some functional forms $b\left(Y^{A}\right)$ for which the quadratic constraints $d o$ hold, let's note two final points. First, by design, the forms (3.4) are consistent with the requirement $S_{A} \rightarrow 0$ as $Z^{A} \rightarrow 0$, which is a standard gauge choice. Second, we could generalize (3.4) without spoiling the holomorphy conditions or this standard gauge choice by adding to $s_{2}$ a function only of $z^{2}$ and $Y^{A}$ which vanishes as $z^{2} \rightarrow 0$; and likewise we could add to $\bar{s}_{\dot{2}}$ a function of $\dot{z}^{2}$ and $Y^{A}$ which vanishes as $\bar{z}^{\dot{2}} \rightarrow 0$. We will not consider such generalizations in this paper, but instead restrict ourselves to (3.4) as written. 
The simplest non-trivial solution to (3.3)-(3.4) is

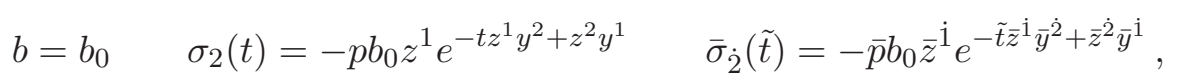

where $b_{0}$ is a constant. A stronger, unintegrated form of the quadratic constraints in (3.3) can be shown to hold for this case:

$$
\left\{\sigma_{2}(t), b * K\right\}_{*}=0 \quad\left[\bar{\sigma}_{\dot{2}}(\tilde{t}), b * K\right]_{*}=0 \quad\left[\sigma_{2}(t), \bar{\sigma}_{\dot{2}}(\tilde{t})\right]_{*}=0
$$

for all $t$ and $\tilde{t}$. The second and third of these equations are trivially satisfied because $\sigma_{2}(t)$ and $b * K$ are fully holomorphic in $Y$ and $Z$, while $\bar{\sigma}_{\dot{2}}(\tilde{t})$ is fully anti-holomorphic. The general result (2.9) implies in particular that $K$ anti-commutes with $y^{\alpha}$ and $z^{\alpha}$; so it is easy to see that it anti-commutes with $\sigma(t)$ as written in (3.5). The case of constant $b$ case studied previously in [3]. There however the authors imposed an $\mathrm{SO}(3,1)$ symmetry, which lead to the constraint $s_{\alpha}=z_{\alpha} s(u)$ where $u=y^{\alpha} z_{\alpha}$ and $s(u)$ was expressed as an integral transform of confluent hypergeometric functions. It is not clear to us that the solution of [3] is gauge-equivalent to ours.

An interesting generalization of the constant $b$ solution is

$$
b=Q e^{q_{A B} Y^{A} Y^{B}}+R e^{r_{A B} Y^{A} Y^{B}}
$$

where the only non-vanishing components of $q_{A B}$ and $r_{A B}$ are those with $A$ and $B$ taking values in $\{1, \dot{1}\} . Q, R$, and the non-zero components of $q_{A B}$ and $r_{A B}$ are parameters of the solution. Straightforward but tedious computations suffice to show that the unintegrated constraints (3.6) are satisfied. The importance of being able to take linear combinations of these special Gaussian solutions is that we need not stop at two terms: we can take arbitrarily many, or an integral of infinitely many. In short, any function

$$
b=b\left(\left(y^{1}\right)^{2}, y^{1} \bar{y}^{\mathrm{i}},\left(\bar{y}^{\mathrm{i}}\right)^{2}\right)
$$

together with $s_{2}$ and $\bar{s}_{\dot{2}}$ as specified in (3.4), provides a solution of the equations (2.12). A commonly imposed projection condition on field configurations restricts to functions $B$ which are invariant under sending $y \rightarrow i y$ and $\bar{y} \rightarrow-i \bar{y}$. In the presence of this requirement, which is related to requiring only even integer spins in the full theory, $B$ must be a function of $\left(y^{1}\right)^{4}, y^{1} \bar{y}^{\mathrm{i}}$, and $\left(\bar{y}^{\mathrm{i}}\right)^{4}$.

Another interesting generalization of the constant $b$ solution is

$$
b=Q e^{q_{\alpha \dot{\beta}} y^{\alpha} \bar{y}^{\dot{\beta}}}
$$

where $Q$ and the $q_{\alpha \dot{\beta}}$ are parameters. As before, the unintegrated constraints (3.6) are satisfied once one imposes (3.4). A caveat on solutions of the form (3.9) is that if $\operatorname{det} q_{\alpha \dot{\beta}}$ is a real number less than or equal to -1 then some of the requisite star products are ill-defined, so the status of the solution is less clear. There appears to be no general superposition principle for solutions of the form (3.9) analogous to (3.7). 


\section{Gauge transformations and a mass deformation}

A trivial solution to Vasiliev's equations is $w=s=b=0$. The $A d S_{4}$ solution, which we review in section 4.1, is gauge equivalent to this trivial solution. We go on in section 4.1 to explain in how to apply the same gauge transformation to other solutions starting in the $w=0$ gauge. We then work out a particular example in section 4.2 in which $B \propto \zeta e^{y^{1} \bar{y}^{1}-y^{2} \bar{y}^{2}}$, where $\zeta$ is the radial coordinate in the Poincaré patch of $A d S_{4}$. This example is interesting because the $B$ dependence just mentioned is, in the linearized theory, associated with a massive deformation of the $O(N)$ model.

\subsection{The spacetime connection}

Let's review how the spacetime metric and spin connection are packaged into the spatial components $W$ of the higher spin gauge field $A$. Starting from the vierbein $e^{m}=e_{\mu}^{m} d x^{\mu}$ and spin connection $\omega_{m n}=\omega_{\mu m n} d x^{\mu}$, we define

$$
e_{\alpha \dot{\beta}}=\frac{1}{2 L} e^{m} \sigma_{m \alpha \dot{\beta}} \quad \omega_{\alpha \beta}=\frac{1}{2} \omega_{m n} \sigma_{\alpha \beta}^{m n} \quad \bar{\omega}_{\dot{\alpha} \dot{\beta}}=-\frac{1}{2} \omega_{m n} \bar{\sigma}_{\dot{\alpha} \dot{\beta}}^{m n}
$$

and

$$
e=\frac{1}{2} e_{\alpha \dot{\beta}} y^{\alpha} \bar{y}^{\dot{\beta}} \quad \omega=\frac{1}{4} \omega_{\alpha \beta} y^{\alpha} y^{\beta}+\frac{1}{4} \bar{\omega}_{\dot{\alpha} \dot{\beta}} \bar{y}^{\dot{\alpha}} \bar{y}^{\dot{\beta}}
$$

We have defined

$$
\begin{aligned}
\sigma_{\alpha \dot{\beta}}^{m} & =(\mathbf{1}, \vec{\sigma}) & \bar{\sigma}^{m \dot{\alpha} \beta} & =(\mathbf{1},-\vec{\sigma}) \\
\sigma_{\alpha}^{m n}{ }_{\alpha}^{\beta} & =\frac{1}{4}\left(\sigma_{\alpha \dot{\gamma}}^{m} \bar{\sigma}^{n \dot{\gamma} \beta}-\sigma_{\alpha \dot{\gamma}}^{n} \bar{\sigma}^{m \dot{\gamma} \beta}\right) & \bar{\sigma}_{\dot{\beta}}^{m n \dot{\alpha}} & =\frac{1}{4}\left(\bar{\sigma}^{m \dot{\alpha} \gamma} \sigma_{\gamma \dot{\beta}}^{n}-\bar{\sigma}^{n \dot{\alpha} \gamma} \sigma_{\gamma \dot{\beta}}^{m}\right),
\end{aligned}
$$

where $\vec{\sigma}$ are the usual Pauli matrices. We express $A d S_{4}$ in Poincaré patch coordinates:

$$
e_{(0)}^{m}=\delta_{\mu}^{m} \frac{L}{\zeta} d x^{\mu}
$$

with

$$
\omega_{t \zeta}^{(0)}=\frac{d t}{\zeta} \quad \omega_{x^{1} \zeta}^{(0)}=-\frac{d x^{1}}{\zeta} \quad \omega_{x^{2} \zeta}^{(0)}=-\frac{d x^{2}}{\zeta}
$$

and all other components of the spin connection vanishing except as required by the antisymmetry condition $\omega_{m n}=-\omega_{n m}$. It is straightforward to check that

$$
W_{(0)}=e_{(0)}+\omega_{(0)}
$$

satisfies the higher spin equations of motion with $S=B=0$ : that is,

$$
d W_{(0)}+W_{(0)} * W_{(0)}=0 .
$$

In order to produce a more interesting solution of the equations of motion (2.2), we are going to to gauge transform one of our $w=0$ solutions. Starting with a configuration 
$(a, b)$ of higher spin fields, the general gauge transformation to another configuration $(A, B)$ takes the form

$$
d+A=g^{-1} *(d+a) * g \quad B=g^{-1} * b * \pi(g),
$$

where $g$ is a function of $x^{\mu}, Y^{A}$, and $Z^{A}$. A more explicit form of the transformation of the gauge fields is

$$
W=g^{-1} * d_{x} g+g^{-1} * w * g \quad S=g^{-1} * d_{Z} g+g^{-1} * s * g .
$$

Our focus will be to set $w=0$.

The flatness of $W_{(0)}$ indicates that the $A d S_{4}$ solution is related to the trivial solution $w_{(0)}=0, s_{(0)}=0, b_{(0)}=0$ by a gauge transformation. For $\left(t, x^{1}, x^{2}\right)=(0,0,0)$, the gauge function may be represented as

$$
g^{ \pm 1}=L^{ \pm 1} \equiv \frac{4}{\sqrt{\zeta_{0} / \zeta}+2+\sqrt{\zeta / \zeta_{0}}} \exp \left\{\mp \frac{1-\sqrt{\zeta / \zeta_{0}}}{1+\sqrt{\zeta / \zeta_{0}}} \sigma_{\alpha \dot{\beta}}^{\zeta} y^{\alpha} \bar{y}^{\dot{\beta}}\right\}
$$

where $\zeta_{0}$ is a parameter. For a more complete description of this gauge transformation, including the full $x^{\mu}$ dependence, see for example [7].

\subsection{An example}

As an example of the procedure outlined in the previous section, let's consider the solution

$$
\begin{aligned}
b & =b_{0} e^{-\lambda\left(y^{1} \bar{y}^{\dot{1}}-y^{2} \bar{y}^{\dot{2}}\right)} \\
\sigma_{2}(t) & =-p b_{0} z^{1} e^{\left(y^{1}-\lambda \bar{y}^{\dot{2}}\right) z^{2}-t\left(y^{2}-\lambda \bar{y}^{\mathrm{i}}\right) z^{1}} \quad \bar{\sigma}_{\dot{2}}(\tilde{t})=-\bar{p} b_{0} \bar{z}^{\dot{1}} e^{\left(\bar{y}^{\mathrm{i}}-\lambda y^{2}\right) \dot{z}^{2}-\tilde{t}\left(\bar{y}^{\dot{2}}-\lambda y^{1}\right) \bar{z}^{\mathrm{i}}},
\end{aligned}
$$

where $b_{0}$ and $\lambda$ are real parameters. ${ }^{1}$ In making the gauge transformation, we choose $\sigma^{\zeta}=\sigma^{3}=\left(\begin{array}{cc}1 & 0 \\ 0 & -1\end{array}\right)$, and this choice is in some sense "diagonal" with respect to our earlier choice of $s_{2}$ and $\bar{s}_{\dot{2}}$ as the preferred components of the gauge field. Nothing prevents us from making a different choice of $\sigma^{\zeta}$, but the resulting solution would then be more complicated.

The easiest field to pass through the gauge transformation is $B$, and one finds, at $\left(t, x^{1}, x^{2}\right)=(0,0,0)$, that

$$
B=\frac{4 b_{0} \zeta_{0}}{\lambda_{+}^{2} \zeta} e^{-\left(y^{1} \bar{y}^{1}-y^{2} \dot{y}^{2}\right) \lambda_{-}}
$$

where we have defined combinations

$$
\lambda_{ \pm}=1+\lambda \pm(1-\lambda) \zeta_{0} / \zeta
$$

which come up repeatedly after the gauge transformation. We are interested in taking a $\zeta_{0} \rightarrow \infty$ limit, because in this limit $B$ becomes translationally invariant in the boundary

\footnotetext{
${ }^{1}$ The solution (4.11) obeys the projection conditions that complete the characterization of the minimal higher spin theories, provided $b_{0}$ and $\lambda$ are real. In the notation of [10], these projections are $\pi(\bar{\pi}(X))=X$ for $X=W, S$, and $B$, together with $\iota_{+}(W)=-W, \iota_{+}(S)=-S$, and $\iota_{-}(B)=B$, where $\iota_{ \pm}$are linear maps which reverse the order of star products and send $(y, \bar{y}, z, \bar{z}, d z, d \bar{z}) \rightarrow(i y, \pm i \bar{y},-i z, \mp i \bar{z},-i d z, \mp i d \bar{z})$.
} 
directions. (Another way to put this is that boundary variation of $B$ takes place over a length scale $\Delta x \sim \zeta_{0}$, and we are taking that length scale to infinity.) The specific limit we will consider is $\epsilon \rightarrow 0$ where

$$
\lambda=1-2 \epsilon \quad \zeta_{0}=\frac{1}{\epsilon^{2}}
$$

with $b_{0}$ held constant. Passing (4.12) through this limit, we find

$$
B=b_{0} \zeta e^{y^{1} \bar{y}^{\dot{1}}-y^{2} \bar{y}^{2}} .
$$

The scalar field in the higher spin theory is

$$
\left.\phi \equiv B\right|_{Y^{A}=0}=b_{0} \zeta .
$$

The spinor part of the gauge field may be expressed as

$$
S_{2}=\int_{0}^{1} d u \Sigma_{2}(u)
$$

where

$$
\Sigma_{2}(u)=\frac{d t}{d u} L^{-1} * \sigma_{2}(t) * L,
$$

and $u=u(t)$ is a conveniently chosen integration variable, with $u(0)=0$ and $u(1)=1$. In the present case, a convenient definition is

$$
u=\frac{t \lambda_{+}}{2(1-t) \sqrt{\zeta_{0} / \zeta}+t \lambda_{+}},
$$

because then one finds

$$
\Sigma_{2}(u)=-\frac{4 p b_{0} \zeta_{0} / \zeta}{\lambda_{+}^{2}} z^{1} \exp \left\{\left(y^{1}-\frac{\lambda_{-}}{\lambda_{+}} \bar{y}^{2}\right) z^{2}-u\left(y^{2}-\frac{\lambda_{-}}{\lambda_{+}} \bar{y}^{\mathrm{i}}\right) z^{1}\right\} .
$$

Similar expressions can be found for $\bar{S}_{\dot{2}}=\int_{0}^{1} d \tilde{u} \bar{\Sigma}_{\dot{2}}(\tilde{u})$. As before, these expressions are valid only at $\left(t, x^{1}, x^{2}\right)=(0,0,0)$; however, we may impose (4.14) and pass to the $\epsilon \rightarrow 0$ limit to obtain the translationally invariant expressions

$$
\Sigma_{2}(u)=-p b_{0} \zeta z^{1} e^{-u\left(y^{2}+\bar{y}^{1}\right) z^{1}+\left(y^{1}+\bar{y}^{2}\right) z^{2}} \quad \bar{\Sigma}_{\dot{2}}(\tilde{u})=-\bar{p} b_{0} \zeta \bar{z}^{\dot{1}} e^{-\tilde{u}\left(\bar{y}^{2}+y^{1}\right) \bar{z}^{1}+\left(\bar{y}^{1}+y^{2}\right) \bar{z}^{2}} .
$$

It is possible to check directly that the full equations of motion (2.2) are satisfied when we set

$$
\begin{aligned}
& B=b_{0} \zeta e^{y^{1} \bar{y}^{1}-y^{2} \bar{y}^{\dot{2}}} \quad W=W_{(0)} \\
& S_{1}=\bar{S}_{\dot{1}}=0 \quad S_{2}=\int_{0}^{1} d u \Sigma_{2}(u) \quad \bar{S}_{\dot{2}}=\int_{0}^{1} d \tilde{u} \bar{\Sigma}_{\dot{2}}(\tilde{u})
\end{aligned}
$$

with $\Sigma_{2}(u)$ and $\bar{\Sigma}_{\dot{2}}(\tilde{u})$ as given in $(4.21)$, and with the $A d S_{4}$ connection $W_{(0)}$ as defined in (4.6). However, there is an important subtlety: star products of $\Sigma_{2}(u)$ with $B$, which 
come up in the $D_{z^{2}} B=0$ component of the equations of motion, formally diverge once one has passed to the translationally invariant limit; however, if one replaces $\Sigma_{2}(u)$ by $\left.\Sigma_{2}(t, u) \equiv \Sigma_{2}(u)\right|_{z^{2} \rightarrow t z^{2}}$, then $D_{z^{2}} B$ is proportional to $\left\{\Sigma_{2}(t, u), B * K\right\}_{*}$, which vanishes identically. A similar regulator is needed in order to check the equation $D_{\bar{z}^{2}} B=0$. The other equations of motion can be handled without recourse to this type of regulator. We caution that in other gauges, field configurations involving projectors such as $e^{y^{1} \bar{y}^{\mathrm{i}}-y^{2} \bar{y}^{2}}$ often lead to divergences, for instance in $F_{z^{1} z^{2}}$, which do not cancel. Thus it is challenging to find a solution analogous to (4.22) in a covariant gauge.

The solution (4.22) is interesting because in a linearization around $A d S_{4}$, the natural interpretation of the scalar profile (4.15) and (4.16) is that one is deforming the dual $O(N)$ field theory by a constant mass term for the $N$-dimensional vector $\vec{\phi}$ : to see this, compare the scalar profile to the bulk-to-boundary propagators discussed, for example, in [11-13]. Once we introduce the spinorial connection based on (4.21), we obtain an exact generalization to the full non-linear equations of motion. It is tempting to characterize this solution as a holographic dual of the massive $O(N)$ model. However, caution is in order, because we do not fully understand how the explicit breaking of Lorentz symmetry inherent in our gauge choice $S_{1}=\bar{S}_{\mathrm{i}}=0$ affects the holographic interpretation. Certainly it complicates the usual method $[14,15]$ of extracting a privileged spacetime metric. ${ }^{2}$

\section{Conclusions}

The ansatz (3.2) in axial gauge significantly simplifies the equations of Vasiliev's higher spin theories in four dimensions, leading to a broad class of solutions for $b$ depending only on $y^{1}$ and $\bar{y}^{i}$. Privileging one component of a spinor over the other is in some settings related to picking out a null direction. To see this, recall the equivalence of vectors and bispinors: $v_{\alpha \dot{\beta}}=v_{m} \sigma_{\alpha \dot{\beta}}^{m}$. If we choose, for example, $v_{m}=(1,0,0,1)$, then $v_{\alpha \dot{\alpha}} y^{\alpha} \bar{y}^{\dot{\alpha}}=2 y^{1} \bar{y}^{\dot{1}}$, showing that $y^{1}$ and $\bar{y}^{1}$ have been privileged over $y^{2}$ and $\bar{y}^{2}$. Thus it is a reasonable guess that the solutions where $b=b\left(\left(y^{1}\right)^{2}, y^{1} \bar{y}^{\mathrm{i}},\left(\bar{y}^{\mathrm{i}}\right)^{2}\right)$ are related to shock waves, or to metrics expressed in terms of an Eddington-Finkelstein coordinate. We hope to report further on this class of solutions in the future.

In a more limited but interesting class of solutions, $b$ depends on all four $Y^{A}$ variables, but only through the Gaussian expression given in (3.9). We have explained how a simple special case, $b \propto e^{-\lambda\left(y^{1} \bar{y}^{1}-y^{2} \bar{y}^{2}\right)}$, can be endowed with spacetime dependence through a gauge transformation. In a suitable limit, this special case provides an exact solution improving upon the linearized description of a uniform mass deformation of the planar $O(N)$ model; note however that a cancellation of divergences is required in order to verify the $D B=0$ equation. It would clearly be of interest to compute two-point correlators in this higher spin geometry. If indeed its interpretation as a dual of the massive $O(N)$ model is correct, then correlators should have a Lorentz invariant spectral weight with a continuum of states above a gap. Additional solutions of the full Vasiliev equations (2.2) might be constructed in a similar spirit; in particular, it is reasonable to suspect that an

\footnotetext{
${ }^{2}$ We thank S. Didenko for a discussion on this point.
} 
exact axial gauge solution might be available in which the spatial part of the connection $W$ is the same as for $A d S_{4}$, while the profile of the scalar master field $B$ is the $A d S_{4}$ bulk-to-boundary propagator.

Also important for future work is to generalize the Lorentz covariant treatment of the background metric to situations where as a matter of gauge choice one introduces parameters that break Lorentz symmetry. Our gauge choice is of this type since it can be expressed as $\ell^{\alpha} S_{\alpha}=0=\bar{\ell}^{\dot{\alpha}} \bar{S}_{\dot{\alpha}}$ where $\ell^{\alpha}=\left(\begin{array}{l}1 \\ 0\end{array}\right)=\bar{\ell}^{\dot{\alpha}}$, contrasting with the Lorentzsymmetric condition $z^{\alpha} S_{\alpha}=0=\bar{z}^{\dot{\alpha}} \bar{S}_{\dot{\alpha}}$ studied in previous works such as [14, 15].

\section{Acknowledgments}

We are grateful to S. Giombi for helpful discussions and especially to the referee for useful comments that led to a revision of section 4. This work was supported in part by the Department of Energy under Grant No. DE-FG02-91ER40671.

Open Access. This article is distributed under the terms of the Creative Commons Attribution License (CC-BY 4.0), which permits any use, distribution and reproduction in any medium, provided the original author(s) and source are credited.

\section{References}

[1] M.A. Vasiliev, Consistent equation for interacting gauge fields of all spins in (3+1)-dimensions, Phys. Lett. B 243 (1990) 378 [inSPIRE].

[2] M.A. Vasiliev, More on equations of motion for interacting massless fields of all spins in (3+1)-dimensions, Phys. Lett. B 285 (1992) 225 [inSPIRE].

[3] E. Sezgin and P. Sundell, An exact solution of $4 D$ higher-spin gauge theory, Nucl. Phys. B 762 (2007) 1 [hep-th/0508158] [InSPIRE].

[4] V.E. Didenko and M.A. Vasiliev, Static BPS black hole in $4 D$ higher-spin gauge theory, Phys. Lett. B 682 (2009) 305 [Erratum ibid. B 722 (2013) 389] [arXiv:0906.3898] [INSPIRE].

[5] C. Iazeolla and P. Sundell, Biaxially symmetric solutions to 4D higher-spin gravity, J. Phys. A 46 (2013) 214004 [arXiv:1208.4077] [INSPIRE].

[6] C. Iazeolla, E. Sezgin and P. Sundell, Real forms of complex higher spin field equations and new exact solutions, Nucl. Phys. B 791 (2008) 231 [arXiv:0706.2983] [INSPIRE].

[7] S. Giombi and X. Yin, Higher spins in AdS and twistorial holography, JHEP 04 (2011) 086 [arXiv: 1004.3736] [INSPIRE].

[8] I.R. Klebanov and A.M. Polyakov, AdS dual of the critical $O(N)$ vector model, Phys. Lett. B 550 (2002) 213 [hep-th/0210114] [INSPIRE].

[9] E. Sezgin and P. Sundell, Holography in $4 D$ (super) higher spin theories and a test via cubic scalar couplings, JHEP 07 (2005) 044 [hep-th/0305040] [INSPIRE].

[10] S. Giombi and X. Yin, The higher spin/vector model duality, J. Phys. A 46 (2013) 214003 [arXiv:1208.4036] [INSPIRE]. 
[11] S. Giombi and X. Yin, Higher spin gauge theory and holography: the three-point functions, JHEP 09 (2010) 115 [arXiv:0912.3462] [INSPIRE].

[12] M.A. Vasiliev, Holography, unfolding and higher-spin theory, J. Phys. A 46 (2013) 214013 [arXiv: 1203.5554] [INSPIRE].

[13] V.E. Didenko and E.D. Skvortsov, Exact higher-spin symmetry in CFT: all correlators in unbroken Vasiliev theory, JHEP 04 (2013) 158 [arXiv: 1210.7963] [INSPIRE].

[14] M.A. Vasiliev, Higher spin gauge theories: star product and AdS space, hep-th/9910096 [INSPIRE].

[15] E. Sezgin and P. Sundell, Analysis of higher spin field equations in four-dimensions, JHEP 07 (2002) 055 [hep-th/0205132] [INSPIRE]. 